\title{
Le développement du commerce équitable peut-il conduire à une réduction des injustices?
}

\author{
Une réflexion à partir du cas du café
}

Franck Galtier ${ }^{1}$

Virginie Diaz Pedregal ${ }^{2}$

${ }^{1}$ Cirad UMR MOISA

73 rue Jean-François Breton

TA C-99/15

34398 Montpellier Cedex 5

France

<franck.galtier@cirad.fr>

${ }^{2}$ Gret - Direction Scientifique

Campus du Jardin tropical de Paris 45 bis, avenue de la Belle Gabrielle 94736 Nogent-sur-Marne

France

<diaz@gret.org>
Tirés à part : V. Diaz-Pedregal

\begin{abstract}
Résumé
Le commerce équitable et, plus généralement, les standards sociaux, connaissent ces dernières années un succès grandissant. Leur développement est à la fois quantitatif (augmentation de la part de marché de ces labels) et qualitatif (extension des critères pris en compte). Ceci ne va pas sans tensions, l'extension quantitative se faisant en grande partie à travers les standards "light ", tandis que l'extension qualitative est plutôt le fait des acteurs traditionnels du commerce équitable. Le développement de ces standards peut-il conduire à une réduction des injustices ? Cet article mobilise les principales théories de la justice pour explorer cette question. Il s'appuie plus particulièrement sur le cas du café. Il résulte de l'analyse que si la théorie rawlsienne s'avère clairement en faveur de l'extension quantitative et qualitative du commerce équitable et des standards sociaux, les théories alternatives (libertarienne, utilitariste, marxiste) sont plus réservées. C'est, in fine, à l'ouverture et à la modestie que convie cette réflexion, dans la mesure où l'équité peut être " justement " appréhendée de diverses façons.
\end{abstract}

Mots clés : café ; commerce équitable ; inégalité ; Rawls ; théories de la justice.

Thèmes : économie et développement rural ; productions végétales ; systèmes agraires.

\section{Abstract}

\section{Can the development of Fair Trade improve justice? Some insights from the coffee case}

In recent years, Fair Trade, and more generally social standards, have been very successful. Their development has been quantitative (their market share has increased) but also qualitative (more criteria are taken into account). This development generates certain conflict, as quantitative extension is mainly due to "light" standards whereas qualitative extension stems from traditional actors of Fair Trade. Can such a development of these standards lead to a reduction in injustice? This article uses the main theories of justice to tackle this question. It refers more specifically to the coffee case. According to the Rawlsian theory, the quantitative and qualitative extension of Fair Trade and social standards can clearly improve justice. Still, the alternative theories (Libertarian, Utilitarian, and Marxist) are not so enthusiastic. Finally, this reflexion advocates openness and humility, as fairness can be approached from different points of view.

Key words: coffee; fair trade; inequality; Rawls; theories of justice.

Subjects: economy and rural development; farming systems; vegetal productions. e commerce équitable, et plus généralement les standards sociaux, connaissent ces dernières années un succès grandissant. Prenant des formes très diverses selon leurs promoteurs
(Nicholls et Opal, 2005), ils répondent à des attentes variées de la part des producteurs (Raynolds et al., 2007) comme des consommateurs (OzcaglarToulouse et al., 2006). 
Le développement de ces labels sociaux peut-il conduire à une réduction des injustices? Une telle réflexion se justifie par la rareté de la littérature sur ce thème. En effet, si l'impact du commerce équitable sur le développement local ou la pauvreté a fait l'objet de nombreuses analyses (Osterhaus, 2006), sa contribution à la réduction des injustices a été peu étudiée (Diaz Pedregal, 2007). Les approches en termes de développement local ou de réduction de la pauvreté sont plus courantes (Osterhaus, 2006). Or, le commerce équitable se revendique comme "juste" dans son appellation aussi bien en français qu'en espagnol ("comercio justo") ou en anglais ("fair trade"). Ceci incite à questionner sa contribution à la justice. Dès lors, deux orientations sont possibles : la première consiste à étudier les positions des acteurs du commerce équitable (producteurs, certificateurs, distributeurs, consommateurs, etc.) par rapport à leur conception de la justice, en analysant leurs pratiques et leurs représentations (Béji-Bécheur et al., 2008). Malgré son intérêt, cette approche prête le flan à la critique qu'Amartya Sen adresse aux approches "subjectivistes " : les acteurs peuvent avoir une conception très parcellaire de la justice et être victimes d'une injustice sans en avoir conscience (Sen, 1999). La seconde orientation, choisie par les auteurs de cet article, est encore inédite. Elle consiste à évaluer le développement du commerce équitable et des autres standards sociaux à l'aide de théories de la justice indépendantes des personnes impliquées dans l'action. La théorie de la justice de John Rawls (1997), point de référence dans le monde académique, constitue le pilier de cette réflexion. D'autres théories alternatives de la justice (théories libertarienne, utilitariste et marxiste) serviront de contrepoint à la première conception.

Par " développement du commerce équitable ", nous nous référons à son extension à la fois quantitative et qualitative. L'extension quantitative désigne les gains de part de marché du commerce équitable et des autres standards sociaux. L'extension qualitative renvoie à l'augmentation des critères pris en compte. $\mathrm{Si}$, à son origine, le commerce équitable visait à améliorer prioritairement le sort des "petits producteurs défavorisés" notamment par un accès au crédit et un prix minimum garanti -, il se préoccupe aujourd'hui explicitement des conditions de vie des journaliers et des employés des petites et grandes plantations (FLO, 2007).
Nous illustrerons notre propos par l'exemple du café. Ce produit est vraiment symptomatique du développement du commerce équitable, notamment dans son aspect quantitatif. En effet, si la part de marché des cafés certifiés par le commerce équitable ou d'autres standards sociaux est encore très faible - de l'ordre de $1 \%$ des volumes échangés dans le monde -, elle est nettement à la hausse depuis une quinzaine d'années (FLO, 2007). En outre, elle atteint des niveaux spectaculaires dans certains pays (près de $30 \%$ aux Pays-Bas), ce qui préfigure peut être la place que les cafés sociaux sont appelés à occuper dans le futur. Enfin, une initiative internationale réunissant les principaux acteurs de la filière a vu le jour : le Common Code for the Coffee Community ou 4C. Le 4C a défini des standards sociaux ayant vocation à s'appliquer à la majorité des lots de café verts échangés sur le marché international.

Cette dynamique de développement à la fois quantitatif et qualitatif du commerce équitable est-elle de nature à réduire les injustices ? Telle est la question à laquelle ce papier tentera de répondre. Nous considérerons, dans un premier temps, les différentes formes de l'extension du commerce équitable et des standards sociaux, puis nous les passerons au crible des théories de la justice.

\section{L'extension du commerce équitable et des standards sociaux}

Le commerce équitable, tel que nous l'entendons aujourd'hui, est apparu à la fin des années 1940 (Nicholls et Opal, 2005). Il reposait alors exclusivement sur les relations personnalisées entre des coopératives de producteurs et des magasins spécialisés dans les pays industrialisés. Dans les années 1980 est apparue la version "certifiée " du commerce équitable, avec le "label " Max Havelaar (Roozen et Van der Hoff, 2002). Par la suite, d'autres labels sociaux se sont développés. Le fait que le café soit rapidement devenu le produit " phare " du commerce équitable, en termes de visibilité et de volumes vendus, n'est pas un fait anodin. Outre la simplicité de la transformation industrielle du produit et sa consommation courante dans les pays occidentaux, le café s'accompagne d'un vaste imaginaire
(Tulet et al., 1994). Il est surtout associé à une histoire sociale mouvementée. Dès lors, il convient d'analyser les principaux problèmes sociaux liés à la production et à la commercialisation du café, afin de comprendre comment le commerce équitable a pu se développer à travers ce produit.

\section{Contexte : les principaux problèmes sociaux liés à la production et à la commercialisation du café}

Dès son introduction dans les Antilles au XVIII ${ }^{\mathrm{e}}$ siècle, la culture du café a reposé sur l'esclavage. C'est en effet pour développer la production de café, de coton et de canne à sucre, que des millions d'hommes ont été déportés d'Afrique vers les Amériques. L'abolition progressive de l'esclavage au cours du XIX ${ }^{\mathrm{e}}$ siècle a contraint les planteurs à rechercher de nouvelles formes de mobilisation du travail. En Afrique, le travail forcé s'est parfois substitué à l'esclavage comme modalité d'exploitation de la main-d'œuvre indigène, avant de faire à son tour l'objet d'une condamnation à l'Organisation internationale du travail (OIT) en 1930 (Daviron, 2008). Parallèlement, s'est aussi développée une tentative de constitution d'un salariat agricole (CoOper, 1996). Malgré un soutien de l'administration coloniale au système de la grande plantation - des systèmes de prix discriminatoires en faveur des colons ont parfois été mis en place - (Greenhill, 1995) ${ }^{1}$, l'agriculture familiale s'est imposée comme le mode de production dominant pour le café. Aujourd'hui, $80 \%$ du café produit dans le monde sort des exploitations familiales d'Amérique, d'Afrique ou d'Asie. Les problèmes sociaux liés à la culture du café n'ont pas disparu pour autant. Le salariat agricole reste la règle au sein des grandes plantations, mais existe aussi dans les petites exploitations, où une main-d'œuvre occasionnelle est très souvent employée au moment de la récolte. Or, certains salariés travaillant dans les plantations de café sont particulièrement vulnérables (maind'œuvre infantile, esclave ou immigrée en situation illégale). En outre, le droit du travail n'est pas toujours appliqué : absence

\footnotetext{
${ }^{1}$ C'est le développement des marchés à terme du café dans la deuxième moitié du $X I X^{e}$ siècle qui a permis l'émergence d'acheteurs dans les pays producteurs, levant ainsi le principal blocage à l'apparition de l'agriculture paysanne (Daviron, 2002).
} 
Tableau 1. Part de marchés des cafés équitables et durables dans le monde.

Table 1. Market share of fair trade and sustainable coffees at the world scale.

\begin{tabular}{lccrcr}
\hline & \multicolumn{2}{c}{ Volume } & & \multicolumn{2}{c}{ Valeur } \\
\cline { 2 - 3 } \cline { 5 - 6 } & Milliers de sac de $\mathbf{6 0} \mathbf{~ k g}$ & $\%$ & & Millions de dollars US & \% \\
\hline Café durable & 318 & 0,3 & & 565 & 1,2 \\
Café durable certifié & 273 & 0,2 & & 490 & 1,0 \\
Café du commerce équitable & 221 & 0,2 & 393 & 0,8
\end{tabular}

Source : Daviron et Ponte, 2004.

de contrat de travail formel et de syndicats, et dépendance totale envers le planteur pour le logement et l'approvisionnement en produits de première nécessité sont parfois observées.

Les problèmes sociaux relatifs à la commercialisation du café sont également importants. Ils ont trait à la répartition de la valeur ajoutée au sein de la chaîne globale de valeur. Jusqu'à la fin des années 1980, le marché international du café vert était très encadré. En 1989, l'Accord international sur le café (AIC), qui régulait depuis près de trente ans le marché du café vert par un système de quotas de production, est abandonné. Les marketing boards sont démantelés. Les multinationales du négoce (de moins en moins nombreuses, du fait de forts mouvements de concentration) mettent en place des systèmes d'approvisionnement direct dans les principaux pays producteurs. Le pouvoir du marché passe de leur côté. Le prix du café à la production chute vertigineusement, tandis que le prix du produit torréfié reste stable. En 20022003, le prix international du café atteint son plus bas niveau historique en valeur réelle (environ 40 dollars US par quintal).

\section{Extension quantitative du commerce équitable et autres standards sociaux}

Forme de commerce entièrement " alternative "de 1950 à la fin des années 1970, le commerce équitable s'ouvre au monde de la grande distribution dans les années 1980 par la mise en place d'un système de certification, offrant un certain nombre de garanties aux producteurs et aux consommateurs (Roozen et Van der Hoff, 2002). Les critères développés par Fair trade Labelling Organizations (FLO), principal organisme de certification du commerce équitable, portent sur le fonctionnement des organisations de producteurs et les engagements des acheteurs de café. La codi- fication du commerce équitable sous forme de cahiers des charges précis et détaillés, alliée à la professionnalisation des activités des militants, ont permis la vente des produits équitables en grande distribution. Elles ont conduit à une augmentation des volumes de vente sans précédent dans l'histoire de ce commerce.

Ces dernières années, un nouveau processus est à l'œuvre: des labels sociaux " light", moins exigeants que le commerce équitable traditionnel, font progressivement leur apparition. Utz Kapeh et Rainforest Alliance appartiennent à cette catégorie. À la différence du commerce équitable, ces labels ne proposent pas de prix minimum garanti. La diversification des labels permet a priori de mieux satisfaire la demande, puisque les consommateurs ont des degrés d'exigence et de "consentement à payer " très divers en matière sociale. Cependant, le risque de confusion entre labels est réel pour des consommateurs peu attentifs ou insuffisamment informés.

Néanmoins, les volumes de café vendus aux conditions du commerce équitable et des autres standards sociaux sont encore modestes. En 2004, la part des cafés certifiés équitable est de $0,2 \%$ en volume et de $0,8 \%$ en valeur. Celle de l'ensemble des cafés durables n'est guère plus élevée (tableau 1).

Néanmoins, plusieurs éléments donnent à penser que le commerce équitable et les standards sociaux sont en train de sortir de l'étroite niche de marché dans laquelle ils sont restés cantonnés. En premier lieu, l'exemple des Pays-Bas montre que les cafés certifiés par le commerce équitable ou d'autres standards sociaux peuvent représenter un segment de marché très important. En effet, les cafés "équitables " et "sociaux " constituent aujourd'hui près de $30 \%$ du marché du café de ce pays (figure 1). Les Pays-Bas ont été parmi les pionniers en matière de consommation des produits issus du commerce équitable.
Leur modèle pourrait être repris dans d'autres régions du monde.

Par ailleurs, une initiative internationale portée par une table ronde "multiacteurs "vise à instaurer des critères sociaux ayant vocation à s'appliquer à la majorité des transactions commerciales de café. Cette initiative, le Common Code for the Coffee Community (4C), se présente comme "une association globale visant un développementsocial, environnemental et économique durable dans le secteur conventionnel du café "2. Le 4C a défini, de façon relativement ouverte, un cahier des charges et un système de contrôle et de certification. La plupart des principaux opérateurs (négociants, torréfacteurs, grandes organisations de producteurs, associations d'exportateurs, organismes publics nationaux ou régionaux et instituts de recherche) se sont impliqués dans la démarche, avec un rôle important joué par les multinationales du négoce et de la torréfaction (Neilson et Pritchard, 2007). Le cahier des charges actuel définit des critères sociaux faibles mais ayant vocation à s'appliquer au mainstream market.

\section{Extension qualitative du commerce équitable}

Dans les premières démarches de commerce équitable, les critères à satisfaire portaient essentiellement sur les associations de producteurs qui devaient être ouvertes, transparentes et démocratiques. Pour le reste, les exploitations agricoles étaient considérées comme des "boites noires". On supposait implicitement qu'en améliorant la situation des propriétaires des plantations (par le prix minimum garanti et le crédit), le commerce équitable favoriserait également le sort de l'ensemble des personnes travaillant à la production du café. Cette approche était justifiée par

\footnotetext{
$\overline{{ }^{2} w w w \cdot 4 c}$-coffeeassociation.org.
} 
le fait que les petits producteurs mobilisent essentiellement de la main-d'œuvre familiale.

Un premier pas vers l'extension a été franchi par FLO en mettant en place une certification "grande plantation ", garantissant certains droits pour les travailleurs salariés (niveau de salaire, conditions de travail et de vie). Cette certification ne s'applique cependant qu'à un nombre restreint de produits, comme les bananes, le thé ou les fleurs (mais pas le café). La question des droits des travailleurs des " petites plantations " n'a été abordée que récemment par FLO. Les critères reprennent une partie des normes de l'OIT, appliquées en faveur des travailleurs occasionnels. Une variante plus ambitieuse consisterait à étendre la protection offerte par le commerce équitable à la main-d'œuvre familiale des petites plantations, qui, actuellement, n'est pas soumise à une protection légale.

D'autres conceptions du commerce équitable, comme celle défendue par l'association Minga en France, proposent d'aller plus loin, en étendant le soutien offert par le commerce équitable à l'ensemble des salariés de la filière équitable. Ainsi, les employés des entreprises du transport et de la distribution bénéficieraient également d'une certaine protection économique et sociale, spécifique à l'échange équitable. Pour des raisons de coût et de faisabilité, les démarches entreprises en ce sens sont encore très restreintes.

\section{Des extensions antagoniques?}

Du fait de l'essor des labels "light" (Utz Kapeh, Rainforest Alliance...), de l'initiative 4C et d'une meilleure information des consommateurs, le développement du commerce équitable et des autres standards sociaux devrait se poursuivre, notamment pour la filière café. Ce développement se traduirait non seulement par un accroissement du volume de vente, mais aussi par l'élargissement du spectre des travailleurs couverts. Ces deux dynamiques ne sont pourtant pas nécessairement compatibles : l'extension quantitative des formes alternatives du commerce du café se fait en grande partie à travers la multiplication des standards "light", tandis que l'extension qualitative est plutôt le fait des acteurs entièrement dédiés au commerce équitable, comme FLO et Minga. Or, l'allégement des exigences des labels facilite l'obtention de nouvelles parts de marché. Dès lors, la tentation est grande pour les grandes entreprises de créer des standards sociaux au "rabais" (Becchetti et Huybrechts, 2008). Si les consommateurs ne sont pas bien informés sur les différences entre labels, les labels "light" risquent d'évincer les labels plus exigeants par un phénomène de sélection adverse.

Cette concurrence entre labels se retrouve aux Pays-Bas : tandis que la part de marché des cafés équitables plafonne à $3 \%$, celle des labels "light" atteint $25 \%$ (figure 1). Le succès de Utz Kapeh dans ce pays est

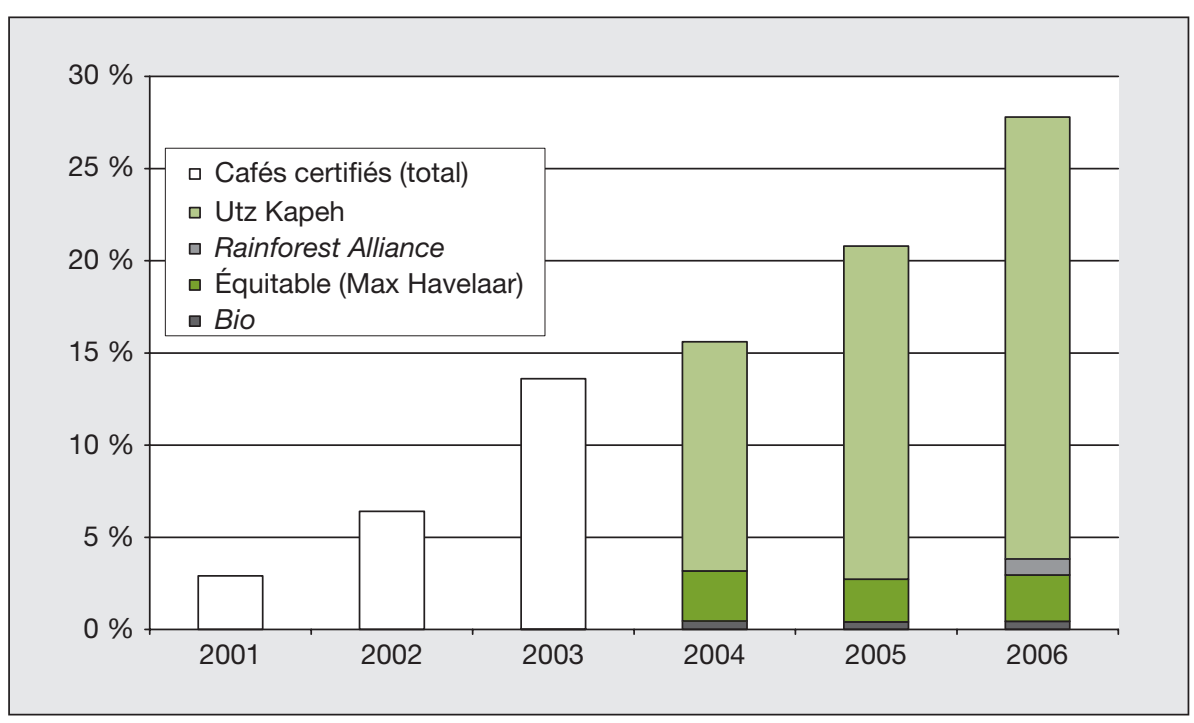

Figure 1. Part de marché des cafés certifiés aux Pays-Bas (2001 - 2006).

Figure 1. Market share of certified coffees in the Netherlands (2001-2006). Source: Dutch Coffee Coalition. en grande partie dû à la promotion du label par les industriels du café, comme Douwe Egberts. De même, les multinationales ont joué un rôle de premier plan dans l'élaboration du label 4C.

L'extension - qualitative et quantitative - du commerce équitable et des autres standards sociaux au cours des prochaines années reste suffisamment probable pour qu'il soit intéressant de l'évaluer en termes de justice. C'est l'objet de la seconde partie de l'article.

\section{Extension}

\section{du commerce équitable et théories de la justice}

Nous considérerons en premier lieu la théorie de la justice de John Rawls, qui occupe actuellement une place dominante dans la réflexion sur la justice sociale. Puis, nous nous attacherons aux principales théories alternatives, en nous fondant, pour chacune d'entre elles, sur un auteur de référence : Robert Nozick pour les libertariens, John Harsanyi pour les utilitaristes et John Roemer pour les marxistes.

\section{Extension du commerce équitable et critères de justice de John Rawls}

Selon le libéral-égalitariste John Rawls (1971), trois types de "biens premiers " peuvent être distingués dans une société : les libertés fondamentales, les chances d'accès aux positions sociales et les avantages socio-économiques liés aux positions sociales. La justice dépend $\mathrm{du}$ respect d'une règle d'allocation spécifique pour chacun de ces types de biens :

- les libertés fondamentales (droit de vote et d'éligibilité, liberté d'expression et de réunion, liberté de conscience et de pensée, liberté de détenir de la propriété personnelle, protection contre l'arrestation et la dépossession arbitraires) sont régies par le principe d'égale liberté, selon lequel toute personne a droit à l'ensemble le plus étendu de libertés fondamentales qui soit compatible avec un ensemble semblable de liberté pour tous ;

- les chances d'accès aux positions sociales sont régies par le principe d'égalité équitable des chances, selon lequel, à égalité de 
talents, toute personne doit avoir les mêmes possibilités d'accès aux différentes positions sociales ;

- les avantages socio-économiques liés aux positions sociales (revenus et richesses, pouvoirs et prérogatives, bases sociales du respect de soi) sont régis par le principe de différence, selon lequel, à égalité de talents, les différences dans la répartition de ces biens doivent bénéficier aux individus les plus défavorisés (principe du maximin).

Comme des conflits peuvent émerger entre les règles d'allocation des différentes catégories de bien, une hiérarchie des règles a été établie. Les libertés fondamentales priment sur les chances d'accès aux positions sociales, elles-mêmes étant prioritaires sur les avantages socio-économiques liés aux positions sociales : c'est ce que Rawls appelle "l'ordre lexicographique".

Dans une perspective rawlsienne, l'esclavage et le travail forcé sont profondément injustes parce qu'ils privent les travailleurs de leurs libertés fondamentales. L'émergence de l'agriculture paysanne et du salariat assure le respect de certaines libertés, mais reste insuffisante, notamment lorsque le droit du travail n'est pas appliqué. En ce sens, le commerce équitable contribue à la réduction des injustices, puisqu'il garantit les droits de certains travailleurs. Cependant, dans sa version actuelle, le commerce équitable est loin de satisfaire le principe d'égale liberté. En effet, les libertés fondamentales garanties par le commerce équitable ne s'appliquent qu'à un nombre restreint de catégories de travailleurs : membres d'associations de producteurs, salariés de grandes plantations (pour certains produits) et, depuis peu, salariés des petites plantations. Elles ne s'appliquent en outre qu'à un faible pourcentage de travailleurs (ceux oeuvrant dans des structures participant au commerce équitable). Le degré de satisfaction du principe d'égale liberté, principe de justice le plus fondamental pour Rawls, serait ainsi fortement augmenté si le commerce équitable était étendu, d'une part, à l'ensemble des catégories de travailleurs, et, d'autre part, à l'ensemble des unités de production de café.

Le premier point renvoie à l'extension qualitative $^{3}$ du commerce équitable ; le second à son extension quantitative. Les deux extensions jouent donc en faveur du principe d'égale liberté.

\footnotetext{
${ }^{3}$ Concrètement, ceci renvoie à l'extension de la protection du commerce équitable à la maind'œuvre familiale et aux salariés des entreprises situées en aval de la filière (transport, torréfaction ou distribution).
}

L'extension du commerce équitable s'avérerait également favorable au principe d'égalité équitable des chances, dans la mesure où elle rendrait ce label accessible à un plus grand nombre de producteurs. Le commerce équitable respecte-t-il le "principe de différence "? On dit parfois que le commerce équitable ne s'adresse pas aux producteurs les plus défavorisés. En effet, les petits producteurs non organisés et les organisations de producteurs les plus faibles, n'ayant pas la capacité d'exporter directement, sont d'emblée exclus de l'accès au système équitable. Cependant, si on considère que les salariés agricoles sont plus défavorisés que les producteurs indépendants, alors l'inclusion croissante des conditions de travail de ces salariés dans les critères du commerce équitable contribue à améliorer la satisfaction du principe de différence. Dès lors, le principe de différence conduit à une recommandation convergente à celle fondée sur les principes d'égale liberté et d'égalité équitable des chances: l'extension du commerce équitable contribuerait à réduire les injustices.

La perspective libertarienne se distinguet-elle de ce schéma ? L'extension du commerce équitable et des standards sociaux apparaît-elle aussi bénéfique que dans l'approche rawlsienne?

\section{La perspective libertarienne}

Selon le libertarien Robert Nozick (1988), la justice d'un acte ou d'une institution n'est pas évaluée en fonction de ses conséquences, mais selon sa conformité à trois principes éthiques, à savoir :

- le principe de propriété de soi, qui stipule que chacun doit avoir la liberté de disposer de son corps à sa guise, sans violer les droits des autres individus. Ceci implique un certain nombre d'autorisations (euthanasie, prostitution, vente d'organes...) et le refus d'obligations (service militaire, ceinture de sécurité...). Être propriétaire de sa propre personne suppose non seulement la propriété de ses aptitudes, mais aussi du fruit de ses aptitudes (comme son travail) ;

- le principe de juste circulation, qui mentionne que les droits de propriété d'une personne sur les objets sont légitimes si l'objet a été acquis par une transaction volontaire et sans fraude ou s'il a été fabriqué par la personne en n'utilisant que des biens auparavant acquis légitimement ; - le principe d'appropriation originelle des ressources naturelles, qui implique qu'une appropriation est légitime dans la mesure où ceux qui en sont privés ne se trouvent pas dans une situation pire que celle qui était la leur avant cette appropriation (auquel cas une compensation peut être exigée).

D'un point de vue libertarien, le commerce équitable peut contribuer à renforcer le respect du principe de propriété de soi. En effet, si des progrès importants ont été réalisés depuis l'abolition de l'esclavage et du travail forcé, le problème n'est pas résolu pour autant. Des violations flagrantes du principe de propriété de soi (absence de consentement du travailleur à accomplir sa tâche, punitions corporelles, etc.) peuvent encore se produire aujourd'hui. En effet, nombre de salariés - ceux des grandes plantations mais aussi la main-d'œuvre occasionnelle employée par les petits producteurs pour la récolte - ne bénéficient pas d'une protection effective par le droit du travail. En outre, le principe de propriété de soi peut facilement être violé pour la maind'œuvre familiale, si le chef de famille abuse de son autorité. Les principes libertariens conduisent donc à considérer d'un œeil bienveillant l'extension qualitative du commerce équitable, c'est-à-dire l'application des critères du commerce équitable à de nouvelles catégories de travailleurs, tels les salariés des grandes plantations de café, la main-d'œuvre familiale des petites plantations, et les salariés des entreprises de transport, de torréfaction ou de distribution.

Dans une perspective libertarienne, le commerce équitable et les standards sociaux ne violent pas le principe de juste circulation puisqu'ils reposent sur le consentement volontaire des différentes parties prenantes (producteurs, torréfacteurs, distributeurs et consommateurs). En revanche, toute tentative de légiférer pour empêcher le développement des labels " light " doit être condamnée. Le principe de juste circulation implique en effet une totale liberté de concurrence, même si certaines mesures, comme l'obligation d'informer les consommateurs, peuvent être nécessaires pour empêcher une confusion qui pourrait s'apparenter à de la fraude. Enfin, toute initiative visant à imposer le commerce équitable doit être elle aussi condamnée, qu'il s'agisse d'en faire supporter les coûts aux acteurs de la filière (comme dans l'initiative 4C) ou aux contribuables (si l'État apporte un soutien financier au commerce équitable).

$\mathrm{Si}$ la pensée libertarienne conduit à considérer positivement l'extension qualitative du commerce équitable, elle est, 
en revanche, plus réservée sur l'extension quantitative de ce commerce. En effet, l'extension quantitative peut impliquer le recours à une forme de contrainte (par des subventions publiques ou l'imposition de l'initiative 4C, par exemple), ce qui serait incompatible avec les principes de justice libertariens. Qu'en est-il à présent du point de vue utilitariste?

\section{Le point de vue utilitariste}

Selon l'utilitariste John Harsanyi (1955), est juste ce qui permet de maximiser le bien-être moyen des individus d'une population donnée.

Comme les libertariens, les utilitaristes sont globalement favorables à la liberté des individus. Cependant, la liberté n'est pas recherchée pour elle-même, mais pour l'accroissement de bien-être qu'elle est supposée générer. Si une activité ou une norme augmente le bien-être moyen, alors elle peut être mise en application sans recourir à la contrainte : il suffit de compenser les perdants. Dans une perspective utilitariste, la liberté permet donc de garantir qu'une activité ou une norme est bénéfique pour la société. Ceci entraîne deux conséquences pour le commerce équitable. En premier lieu, la libre concurrence entre labels doit être respectée, car elle conduit à satisfaire au mieux les préférences de chacun. Par exemple, certains consommateurs sont disposés à payer plus cher pour des labels " exigeants " tandis que d'autres préféreront des labels "light ". Ceci conduit à écarter l'idée d'une réglementation trop stricte du commerce équitable. En second lieu, la participation au système équitable doit dépendre exclusivement des choix individuels, ce qui semble exclure les standards imposées par une action collective (4C) ou par les choix budgétaires de l'État (subventions). D'un point de vue utilitariste, ce n'est pas tellement l'extension qualitative du commerce équitable qui est une bonne chose que la coexistence de labels "light " avec des labels plus exigeants. Quant à l'extension quantitative, elle est souhaitable tant qu'elle ne repose pas sur la contrainte. Si on en restait là, les recommandations des utilitaristes seraient en tous points similaires à celles des libertariens.

Le problème se complique cependant si on prend en compte deux phénomènes. Le premier concerne les asymétries d'information et les problèmes de sélection adverse qu'elles engendrent. La diversification du commerce équitable (certification "petites plantation" et "grandes plantations ", labels exigeants et labels "light") peut générer une confusion pour les consommateurs. Comme nous l'avons déjà mentionné, ceci peut entrâ̂ner une substitution entre labels (les labels " light " ou le 4C faisant disparaitre les labels plus exigeants), voire même une contraction de la demande de café équitable, liée à une perte de confiance dans les labels. Renoncer à la certification "grandes plantations " ou légiférer pour empêcher le développement de labels trop "light" peut alors être la meilleure solution pour maximiser le bien-être des individus.

Le second phénomène concerne l'effet bénéfique de la redistribution sur le bien-être (dans le sens où 1 euro additionnel produit plus de bien-être chez un pauvre que chez un riche). De manière générale, le commerce équitable génère un transfert monétaire des consommateurs des pays riches vers les producteurs des pays pauvres (Raynolds et al., 2007). Ce transfert peut être significatif dans les périodes où les prix internationaux sont bas (figure 2), ce qui justifie alors des mesures visant à développer le commerce équitable, en octroyant par exemple des subventions publiques aux promoteurs de ce commerce.

Ainsi, d'un point de vue utilitariste, le développement du commerce équitable apparaît de façon positive : il permet de mieux satisfaire les préférences des consomma- teurs, demandeurs d'attributs éthiques, tout en assurant une augmentation du bien-être des producteurs dans les pays en développement. Dans sa version la plus simple, l'utilitarisme conduit à porter un jugement assez proche de celui des libertariens : évaluation positive de la multiplication des standards (grandes plantations, labels "light") mais plutôt négative des innovations qui pourraient amoindrir leur aspect volontaire (subventions publiques, 4C). Cependant, en prenant en compte les asymétries d'information et l'effet bénéfique de la redistribution, les conclusions sont plus nuancées. L'approche marxiste apporte un autre regard.

\section{L'approche marxiste}

Selon le marxiste John Roemer (1982), la justice est essentiellement liée à la répartition égalitaire des richesses dans la société: une personne est exploitée dans la mesure où elle se trouverait mieux lotie dans une hypothétique situation d'égalité distributive. Il existe trois sources d'injustice, liées respectivement à l'inégalité des dotations en liberté formelle, à l'inégalité en capital physique ou financier et à celle en capital humain (Roemer, 1982). Une répartition égalitaire des libertés formelles et du capital (physique ou financier) permet d'atteindre le socialisme ("à chacun selon son travail "),

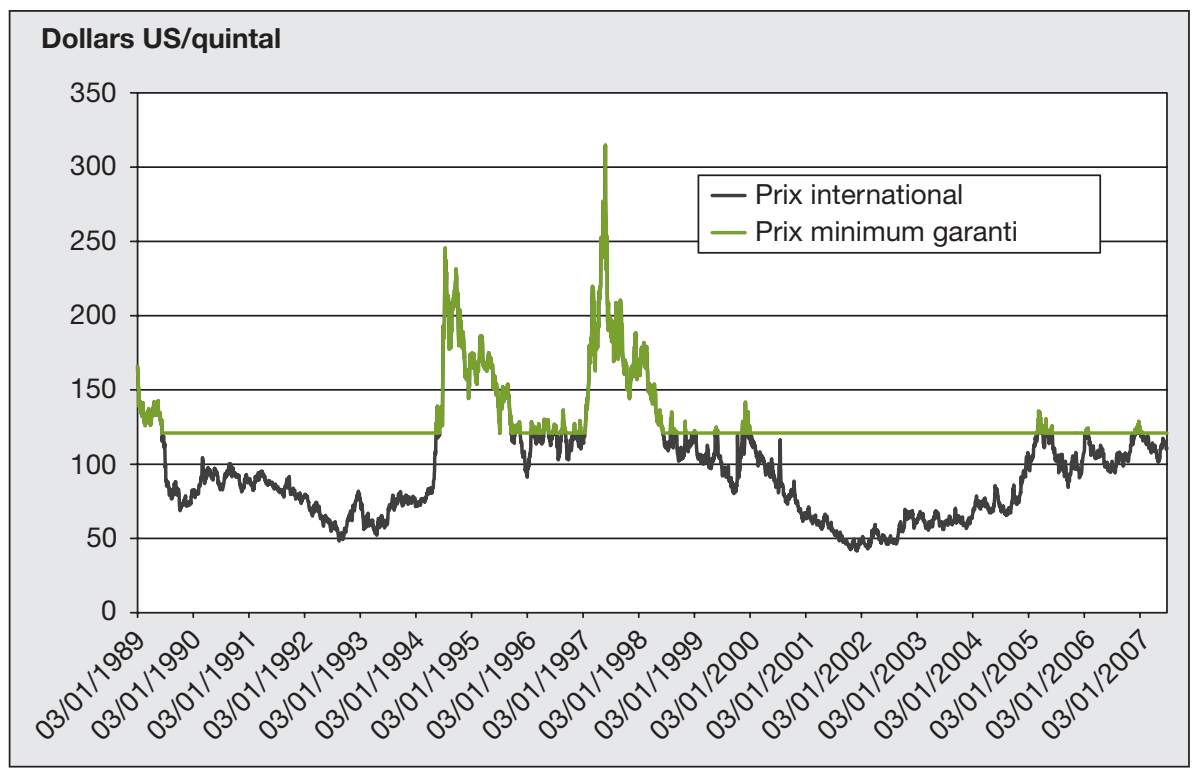

Figure 2. Évolution comparée du prix mondial du café vert et du prix minimum garanti par le commerce equitable.

Figure 2. Comparison of the evolution of world green coffee price and guaranteed minimum price by fair trade.

Sources : NYBOT C Contract, pour le prix international, et FLO, pour le prix minimum garanti. 
tandis que le communisme "à chacun selon ses besoins ") requiert également une compensation des inégalités de dotation en capital humain (talents, santé, âge, handicap, formation...).

En matière de rémunération pour la production de café, les inégalités peuvent être pensées à deux niveaux : au niveau de la distribution de la valeur au sein de la filière (notamment entre pays en développement exportateurs et pays industrialisés importateurs) et au niveau de la distribution de la valeur entre les propriétaires des plantations et leurs salariés.

En ce qui concerne la distribution de la valeur au sein de la filière, l'injustice est surtout liée aux transferts de plus-value des acteurs du Sud (production, exportation) vers les acteurs du Nord (négoce, torréfaction, distribution). Ces transferts résultent d'une répartition inégalitaire du capital (Emmanuel, 1969 ; Amin, 1988). Le seul moyen d'inverser les termes de l'échange consiste à créer un pouvoir de marché en faveur des producteurs. Cet effet a été partiellement atteint par l'Accord international sur le café (AIC). Le caractère injuste du marché concurrentiel, du fait des transferts de plus-value, était en partie compensé par un pouvoir de marché du côté de l'offre, grâce au système des quotas. La fin de l'AIC, le démantèlement des marketing boards et le mouvement de concentration au niveau du négoce et de la torréfaction ont fait passer le pouvoir de marché du côté de la demande. Aujourd'hui, les deux facteurs (transferts de plus-value et pouvoir de marché) jouent dans le même sens: celui d'une concentration de la richesse en aval de la filière. De fait, la part de la valeur ajoutée captée par les pays producteurs est passée de $37 \%$ dans les années 1970, à moins de $16 \%$ dans les années 1990 (Daviron et Ponte, 2004). À l'évidence, le commerce équitable n'est pas parvenu à enrayer la dégradation des termes de l'échange aux dépens des producteurs.

Comment expliquer l'impuissance du commerce équitable dans ce domaine? Si le commerce équitable conduit à une répartition de la valeur ajoutée plus avantageuse aux producteurs que le commerce conventionnel (Nicholls et Opal, 2005), ses effets sont cependant limités par son faible poids dans les échanges internationaux. Dès lors, l'approche marxiste est favorable à l'augmentation de la part de marché du commerce équitable. Néanmoins, l'extension quantitative du commerce équitable passe en grande partie par le développement des standards sociaux "light", dont le prix minimum n'est pas toujours garanti. En ce sens, la réduction des inégalités au niveau international semble compromise. L'approche marxiste conduit donc à approuver la mise en place d'une législation destinée à empêcher le développement des labels "light ". À ce titre, le label $4 \mathrm{C}$, soutenu par des multinationales, est hautement suspect (Neilson et Pritchard, 2007). En revanche, les subventions publiques destinées à étendre la part de marché du commerce équitable, certifié par des labels exigeants, sont considérées comme bénéfiques.

Enfin, il convient d'ajouter que, selon l'approche marxiste, la répartition de la valeur d'un bien entre les planteurs et leurs salariés est par nature injuste, car l'exploitation est intrinsèque au contrat de travail. La seule façon de supprimer cette exploitation est de dépasser le cadre du travail salarié, ce qui a été réalisé en partie par le triomphe de l'agriculture familiale dans les années 1970-1980. Cependant, le commerce équitable n'est pas hostile au salariat, dans la mesure où des journaliers et des permanents peuvent être respectivement employés dans les exploitations familiales et les grandes plantations. En outre, les autres maillons de la filière (transport, torréfaction, distribution) ont presque exclusivement recours au salariat. De ce fait, l'extension qualitative du commerce équitable a un effet ambigu. Si, en améliorant les conditions de travail et de salaire des hommes, elle contribue à une diminution de l'exploitation à court terme, dans le même temps, elle légitime la relation salariale et participe au maintien de l'exploitation à long terme.

\section{Conclusion}

Le développement du commerce équitable peut-il conduire à une réduction des injustices? À travers le cas du café, nous avons, en premier lieu, montré la double dynamique d'extension quantitative et qualitative du commerce équitable. Nous avons vu que cette dualité peut être source de tensions, dans la mesure où l'extension quantitative des formes alternatives de commerce équitable se fait en grande partie à travers la multiplication des standards "light ", tandis que l'extension qualitative est plutôt le fait des initiatives d'acteurs fortement impliqués dans ce commerce, tels FLO, au niveau international, et Minga, en France. Or, l'allègement des exigences des labels facilite l'obtention de nouvelles parts de marché. L'accroissement de la concurrence entre formes "exigeantes " et formes "light " du commerce équitable est à craindre.

Dans un second temps, nous avons évalué le développement du commerce équitable en matière de réduction des injustices à partir des théories de la justice sociale, notamment celle de Rawls. Il est apparu que les trois principes - d'égale liberté, d'égalité équitable des chances, et de différence - de la théorie rawlsienne s'avèrent en faveur de l'extension quantitative et qualitative du commerce équitable. Les approches libertarienne et utilitariste s'accordent volontiers sur l'extension qualitative du commerce équitable, mais sont plus réservées sur l'extension quantitative de ce commerce. Quant à l'approche marxiste, elle conduit à approuver la mise en place d'une législation destinée à empêcher le développement des labels "light". Pour autant, elle ne plébiscite pas sans réserve l'extension qualitative du commerce équitable, dans la mesure où elle légitime la relation salariale.

Ces différents regards des théories de la justice sur le développement du commerce équitable invitent à l'ouverture et à la modestie : l'équité du commerce peut être comprise de diverses façons. Il revient aux certificateurs d'accepter la pluralité des initiatives concurrentes, aux consommateurs de soutenir la démarche qui leur semble la plus juste et aux producteurs de comprendre les intérêts et les enjeux que présente la multiplication de ces nouveaux marchés.

\section{Références}

Amin S. L'Échange inégal et la loi de la valeur. Paris: Anthropos-Economica, 1988.

Becchetti L, Huybrechts B. The Dynamics of Fair Trade as a Mixed-form Market. Journal of Business Ethics 2008; 81: 733-50. DOI: $10.1108 / 17508610810922695$.

Béji-Bécheur A, Diaz Pedregal V, OzcaglarToulouse N. Fair Trade-Just How Fair Are the Exchanges? Journal of Macromarketing 2008 . 28 : 44-52. DOI : $10.1177 / 0276146707311842$

Cooper F. Decolonization and African society: the labor question in French and British Africa. Cambridge: Cambridge University Press, 1996.

Daviron B. Small Farm Production and the Standardization of Tropical Products. Journal of Agrarian Change 2002 ; 2 : 162-84. 
Daviron B. Mobilizing the African native agriculture: the elaboration of an international standard of colonial government. Second European Congress in World and Global History, Dresden, July 2008.

Daviron B, Ponte S. The coffee paradox: commodity trade and the elusive promise of development. London : Zed Books, 2004.

Diaz Pedregal V. Le commerce équitable dans la France contemporaine. Idéologies et pratiques. Paris : L'Harmattan, 2007.

Emmanuel A. L'Échange inégal. Essai sur les antagonismes dans les rapports économiques internationaux. Paris: Maspero, 1969.

FLO International (Fair Trade Labelling Organizations International). Shaping Global Partnerships 2007. Annual Report 2006/2007. Bonn : FLO International, 2007
Greenhill R. State Intervention in the Brazilian Coffee Trade During the 1920s - a Case Study for New Institutional Economics? In : Harriss, Hunter and Lewis, eds. The New Institutional Economics and Third World Development. London : Routledge, 1995.

Harsanyi J. Cardinal welfare, individualistic ethics, and the interpersonal comparison of utility. Journal of Political Economy 1955; 63 : 309-21.

Neilson J, Pritchard B. Green Coffee? The Contradictions of Global Sustainability Initiatives from an Indian Perspective. Development Policy Review 2007 ; 25 : 311-31.

Nicholls A, Opal C. Fair Trade: Market-Driven Ethical Consumption. London: Sage Books, 2005.

Nozick R. Anarchie, État et Utopie. Paris : PUF, Quadrige, 1988.

Osterhaus A. Business unusual: Successes and challenges of fair trade. Brussels: Fair Trade Advocacy Office, 2006.
Ozcaglar-Toulouse $N$, Shiu $E$, Shaw $D$. In Search of Fair Trade: Ethical Consumer Decision-making in France. International Journal of Consumer Studies 2006 ; 30 : 502-14.

Rawls J. Théorie de la Justice. Paris: Seuil, Points Essais, 1997.

Raynolds LT, Murray DL, Wilkinson J. Fair Trade: The Challenges of Transforming Globalization. London, New York: Routledge Press, 2007.

Roemer J. A General Theory of Exploitation and Class. Cambridge (Massachusetts): Harvard University Press, 1982.

Roozen N, Van der Hoff F. L'aventure du commerce équitable. Paris : Lattès, 2002.

Sen A. Development as Freedom. Oxford: Oxford University Press, 1999.

Tulet J C, Charlery B, Bart F, Pilleboue J. Paysanneries du café des hautes terres tropicales. Paris : Karthala, 1994 